\title{
Association of APOE with Parkinson Disease Age-at-Onset in Women
}

\author{
Daniel D. Buchanan BSc(Hons) ${ }^{1}$, Peter A. Silburn FRACP, $\mathrm{PhD}^{3}$, \\ Jonathan A. Prince $\mathrm{PhD}^{2}$, George D. Mellick $\mathrm{PhD}^{1,3}$
}

\begin{abstract}
${ }^{1}$ University of Queensland School of Medicine and Department of Neurology Princess Alexandra Hospital, Brisbane, Australia

${ }^{2}$ Department of Genomics and Bioinformatics, Karolinska Institute, Stockholm, Sweden ${ }^{3}$ Institute for Cell and Molecular Therapies, Griffith University, Brisbane, Australia
\end{abstract}

Author for correspondence:

Associate Professor George D. Mellick

Institute for Cell and Molecular Therapies

Griffith University

Nathan QLD 4111

Australia

\section{Acknowledgements:}

A/Prof Mellick is supported by the Geriatric Medical Foundation of Queensland. This study was supported by Parkinson's Queensland Inc. 


\begin{abstract}
APOE polymorphism has received extensive attention as a risk factor for Parkinson's disease (PD), but findings have been equivocal. Analysis of $A P O E$ variants in an Australian PD case-control sample revealed a robust association between genotype and Age-at-onset $(\mathrm{AAO})$ of $\mathrm{PD}$ in women $(\mathrm{P}=0.0008)$. These data not only further implicate $A P O E$ in $\mathrm{PD}$, but also provide a stark example of the effects that gender may play in complex disorders.
\end{abstract}




\section{INTRODUCTION}

Genetic association of $A P O E$ with $A D$ is one of the few widely recognized examples of common polymorphism contributing to a common illness (1). The possibility of a genetic mechanism underlying neurodegeneration in $\mathrm{AD}$ has led to the investigation of $A P O E$ in numerous diseases, among which Parkinson's disease has been one of the most studied. In contrast to $\mathrm{AD}$ however, data on $\mathrm{APOE}$ in $\mathrm{PD}$ have been controversial suggesting that a genetic effect, if present, is much weaker than for AD. Previous studies have suggested that $\alpha$-synuclein accumulation can be significantly enhanced by the presence of amyloid precursor protein and $A \beta(2)$. Thus factors, including $A P O E$ genotype, that influence the deposition of these molecules in the brain may also alter risk for PD pathology (3). Recently, sib-pair analysis concluded that the E4 allele was probably responsible for the previously reported chromosome 19 linkage peak for PD (4). However, a meta-analysis which included 22 eligible case-control studies investigating APOE and PD revealed no evidence for an association between the E4 allele and PD (5). Interestingly however, a modest positive association between the E2 allele and PD was revealed (5). In the largest single study performed to date, $\mathrm{Li}$ et al, (6), using family based methodologies, reported that the E4 allele increased the risk of PD but intriguingly was also associated with a decreased AAO of PD. Inherent problems in case-control association studies such as population stratification, inappropriate control subjects and underpowered studies may underlie many of the findings for APOE and PD and contribute to the contradictory reports regarding the $\mathrm{E} 4$ allele as a PD risk factor. With these limitations in mind we studied the common APOE gene variant in a relatively large sample of Australian PD patients and controls of European descent. We have considered both PD risk and AAO in 
genetic models, and have also performed stratified analyses of men and women.

\section{METHODS}

Subjects. The details of our Australian case-control cohort have been published previously (7-9). PD cases $(n=422,239$ males, 183 females, average age $=66.95 \pm 9.80$ (SD) yrs, average onset age $=59.98+10.44$ (SD) yrs) were recruited from Movement Disorders clinics. The diagnosis of probable or definite PD was made by a Movement disorders neurologist using standard criteria. Cases were excluded if they: had a diagnosis of dementia or exhibited symptoms suggestive of an alternative form of parkinsonism. Controls $(\mathrm{n}=387,114$ males, 273 females, average age $=64.16+10.83$ (SD) yrs) consisted of 156 patient spouses and 231 volunteers from patient neighbourhoods and community organizations. All subjects were examined by one of the authors (PAS). Patients with known LRRK2 or parkin gene mutations were not included in the analysis. Data pertaining to ethnic background, family history of PD (first-degree relatives with PD) and AAO of PD symptoms were obtained from study subjects via face-to-face interviews using a structured questionnaire. AAO was defined as self-reported age at which the patient initially noticed the appearance of a salient parkinsonian symptom. All subjects were of European Caucasian ancestry.

Genotyping. APOE genotyping was performed using a previously described method including primer sequences (4). All samples were genotyped in duplicate. 
Statistical Analysis. Genotype and allele frequency comparisons were made using 2x2 contingency tables, Fishers exact and Pearson's chi-squared tests, logistic regression analyses, Kaplan-Meier survival plots, ANOVA, FishersPLSD and Hardy-Weinberg equilibrium tests. All probability tests were 2 sided with a level of significance of 0.05 .

\section{RESULTS}

With regards to our case-control sample, there was a significant over-representation of males in the PD group and controls subjects were younger than cases (Table 1).

Potential effects of APOE variants on PD risk were explored using two models. In model 1 we tested possible differences in genotype distributions between cases and controls for E2/E2, E2/E3, E2/E4, E3/E3, E3/E4, and E4/E4 classes (Table 1). In model 2 we compressed genotype groups to two classes consisting of E4 carriers and non-carriers (Table 1). Neither analysis resulted in significant findings. We also performed a stratified analysis of E4-carriers and non-carriers in men and women separately. There was no evidence of association in either group $\left(\chi^{2}=1.6, \mathrm{P}=0.21\right.$ for men and $\chi^{2}=0.65$, $\mathrm{P}=0.42$ for women, respectively).

The distribution of AAO in the PD sample was normal. Average AAO were statistically equivalent for all genotypes in the entire PD set (Table 2). However, the E4 carrier/noncarrier analysis (model 2), identified marginal evidence that E4 carriers had a lower mean AAO (Table 2). Chi-square analysis of all cases stratified on median AAO (60 years) revealed that E4 carriers were marginally over-represented in the early onset group 
$(\mathrm{OR}=1.50,95 \% \mathrm{CI}=0.97-2.33)$; this was more marked in females $(\mathrm{OR}=2.40,95 \% \mathrm{CI}$ $=1.19-4.82$ ).

We used a second order factorial ANOVA to address a possible interaction with sex, focusing on the e4 carrier model (model 2). The sex x genotype interaction term was significant in this analysis $(\mathrm{F} 1,414=5.4, \mathrm{P}=0.02)$. In separate gender analyses, the effect of $\mathrm{E} 4$ carrier status was found to be significant in the female group $(\mathrm{P}=0.0025)$, but not in males $(\mathrm{P}=0.91)$. We also noted a significant effect of genotype on AAO in females $\left(\mathrm{F}_{4,177}=2.8 ; \mathrm{P}=0.027\right)($ Table 2). Post hoc tests (Fishers PLSD) were significant between $\mathrm{E} 2 / \mathrm{E} 3$ and $\mathrm{E} 3 / \mathrm{E} 4$ classes $(\mathrm{P}=0.036)$ and between $\mathrm{E} 3 / \mathrm{E} 3$ and $\mathrm{E} 3 / \mathrm{E} 4$ classes $(\mathrm{P}=0.0027)$. We observed significant main effects upon $\mathrm{AAO}$ of the rs 7412 variant (ie the $\operatorname{Arg} 176 \mathrm{Cys})$ in females $\left(\mathrm{F}_{2,178}=5.6 ; \mathrm{P}=0.0043\right)$, but not in males $\left(\mathrm{F}_{2,232}=\right.$ $0.18 ; \mathrm{P}=0.83)$.

We used Kaplan-Meier analysis to estimate survival functions, and performed tests with both censored control samples (censored for age-at-exam) and excluding controls. In the female sample, results for models 1 and 2 were highly significant $\left(\chi^{2}=13.7, \mathrm{df}=4 ; \mathrm{P}=\right.$ $0.0083)$ and $\left(\chi^{2}=11.3, \mathrm{df}=1 ; \mathrm{P}=0.0008\right)$, respectively (Figure 1). Similar AAO trends were observed for female cases with and without a family history of PD. Exclusion of E2/E4 genotypes from the analysis did not substantially alter the overall result; here female carriers continued to have and earlier AAO $(\mathrm{p}=0.006)$. E2 carriers did not differ to non-carriers in AAO overall or in gender-stratified analyses. 
One of the possible caveats associated with an analysis of APOE upon AAO for any disease is that genotypes have been shown to change with age (thus suggesting longevity effects). We used logistic regression to test for the possible dependence of APOE genotype upon age in the entire control sample. This was not significant $(\mathrm{P}=0.14)$, but did indicate that population frequencies of the E4 allele may be diminishing with age. We note that this model is the equivalent inverse of an ANOVA modeling the dependence of age (provided that age is normally distributed) upon genotype $\left(\mathrm{F}_{1,385}=2.1 ; \mathrm{P}=0.14\right)$ and that the E4 carrier group had a lower mean age $(62.94 \pm 10.54 \mathrm{yrs}$ vs $64.7 \pm 10.93 \mathrm{yrs})$.

\section{DISCUSSION}

In PD, genetic variation in $A P O E$ has been reported to influence both disease risk and AAO however findings have been conflicting. Zareparsi et al, (10) reported a significantly earlier $\mathrm{AAO}$ of $\mathrm{PD}$ in patients with the combined E3/E4 and E4/E4 genotypes compared to the E3/E3 genotype. Maraganore et al, (11) reported that the E2/E3 genotype was associated with a younger AAO in PD. In male subjects we also observed an earlier AAO for E2/E3 genotypes, but this difference was not statistically significant. The data from a large scale meta-analysis by Huang et al, (5) revealed that the E2/E4 genotype was strongly associated with sporadic PD. Interestingly, we also observed a non-significant over-representation of E2/E4 genotypes amongst females with PD (adjusted OR=1.35). The largest independent study to date, performed by Li et al. (6), indicated association of $A P O E$ with both disease risk and with AAO. In the present study, we largely corroborate those latter findings regarding AAO, but with the added dimension that here effects are only evident in women. 
It is possible that our AAO result is merely a chance finding. However, it has been well documented that gender plays an important role in differentiating risk for PD. Moreover, previous studies suggest that gender is an important modifier of the APOE-E4 effect on neurodegenerative pathologies and Alzheimer's phenotype(12, 13). PD has a complex etiology involving interactions between genetic and environmental factors. One might speculate that the E4 effect is manifest more strongly in female PD patients whose risk may be less influenced by environmental determinants. We have previously speculated that factors that increase $\beta$-amyloid and amyloid precursor protein could have effects in PD through their interactions with the $\alpha$-synuclein protein (3). This effect would be more prominent in the absence of other more direct promoters of $\alpha$-synuclein aggregation.

Our broad interpretation of these findings is that possession of the E4 allele lowers ageat-onset for PD, as it does for $\mathrm{AD}$ (12). Indeed, the E4-effect in $\mathrm{AD}$ is also more prominent in females and it may well be that APOE-E4 for a more general risk factor for neurodegeneration per se. Limitations in power, both for this study, and all previous studies restricts any firm indication as to the effects of various diplotype configurations of E2, E3, and E4 alleles. Power also limits further insight into whether effects are exclusive to women, or present in both genders at different levels, with the effect in males beyond the limit of detection.

Studying the determinants of a complex phenotype like PD is not straightforward. There is a requirement to conduct sufficient analyses to corroborate findings, look for 
consistencies and explore interesting leads. However, this is countered by the necessity to avoid indiscriminate testing, in the absence of solid a priori hypotheses and the accompanying risk of revealing spurious chance findings which have no real influence on disease etiology. In this case the importance of APOE genotype in degeneration is well documented and it is only prudent that interactions between gender (and other potential factors) are considered in examinations of PD risk. Previous failures to investigate gender-effects may have resulted in an important risk factor being overlooked.

Finally, while we emphasize a possible gender component to the involvement of APOE in PD, further studies will be needed in increasingly larger samples to examine this and other possible interactions. We encourage the authors of previous studies on $A P O E$ and PD to revisit their published data to explore for an effect modification by gender. 
Table 1. Gender, age, genotype, and E4 carrier status frequencies for PD patients and controls. Odds ratios (OR) with $95 \%$ confidence intervals were calculated using logistic regression modeling adjusting for age, gender, smoking exposure and positive family history of PD.

\begin{tabular}{|l|c|c|c|}
\hline & Controls & PD & \\
\hline Males & 114 & 239 & \\
\hline Females & 273 & 183 & \\
\hline Total & 387 & 422 & $\mathbf{X} 2, \mathrm{P}<0.0001$ \\
\hline & & & \\
\hline Avg age (yrs) & 64.16 & 66.95 & ANOVA, $\mathrm{P}=0.0001$ \\
\hline & 10.83 & 9.80 & \\
\hline Male Avg age (yrs) & & & \\
\hline SD (yrs) & 64.71 & 67.03 & ANOVA, $\mathrm{P}=0.045$ \\
\hline & 11.35 & 9.53 & \\
\hline Female Avg age (yrs) & & & \\
\hline SD (yrs) & 63.93 & 66.85 & ANOVA, P = 0.0037 \\
\hline APOE Genotypes & 10.61 & 10.16 & \\
\hline E3/E3 & & & OR (95\%CI) \\
\hline E2/E2 & 232 & 258 & \\
\hline E4/E4 & 1 & 1 & N.D. \\
\hline E2/E3 & 11 & 13 & $0.77(0.32-1.87)$ \\
\hline E2/E4 & 37 & 51 & $1.27(0.78-2.07)$ \\
\hline E3/E4 & 11 & 9 & $0.99(0.37-2.65)$ \\
\hline Total & 95 & 90 & $0.86(0.60-1.25)$ \\
\hline & 387 & 422 & \\
\hline APOE E4 carrier status & & & \\
\hline non-E4 carriers & & & \\
\hline E4 carriers & 270 & 310 & \\
\hline Total & 387 & 422 & \\
\hline
\end{tabular}


Table 2. Age-at-onset (yrs (SD)) for genotype, and E4 carrier status for PD patients.

\begin{tabular}{|l|r|r|r|}
\hline AAO & PD & Males & Females \\
\hline Genotype & & & N.D. \\
\hline E2/E2 & $59.06(12.00)$ & $57.12(12.89)$ & $60.92(11.02)$ \\
\hline E2/E3 & $59.67(9.64)$ & $66.50(8.70)$ & $54.20(6.72)$ \\
\hline E2/E4 & $60.68(10.13)$ & $60.14(10.07)$ & $61.37(10.22)$ \\
\hline E3/E3 & $58.18(10.68)$ & $60.06(10.34$ & $55.29(10.70)$ \\
\hline E3/E4 & $62.00(8.26)$ & $62.25(9.48)$ & $61.60(6.84)$ \\
\hline E4/E4 & $59.98(10.44)$ & $60.01(10.41)$ & $59.95(10.50)$ \\
\hline Total Avg AAO & & & \\
\hline & & & \\
\hline E4 carrier status & & $59.74(10.53)$ & $61.28(10.33)$ \\
\hline non-E4 carriers & $60.43(10.45)$ & $60.71(10.15)$ & $55.87(10.05)^{2}$ \\
\hline E4 carriers & $58.75(10.34)^{1}$ & & \\
\hline & & &
\end{tabular}

${ }^{1} \mathrm{~F}_{1,416}=3.2 ; \mathrm{P}=0.076$

${ }^{2} \mathrm{~F}_{1,180}=9.4 ; \mathrm{P}=0.0025$ 
Figure 1. Kaplan-Meyer plot showing cumulative proportion of PD patients surviving versus increasing AAO for APOE E4 carrier status (dotted lines represent E4-carriers).

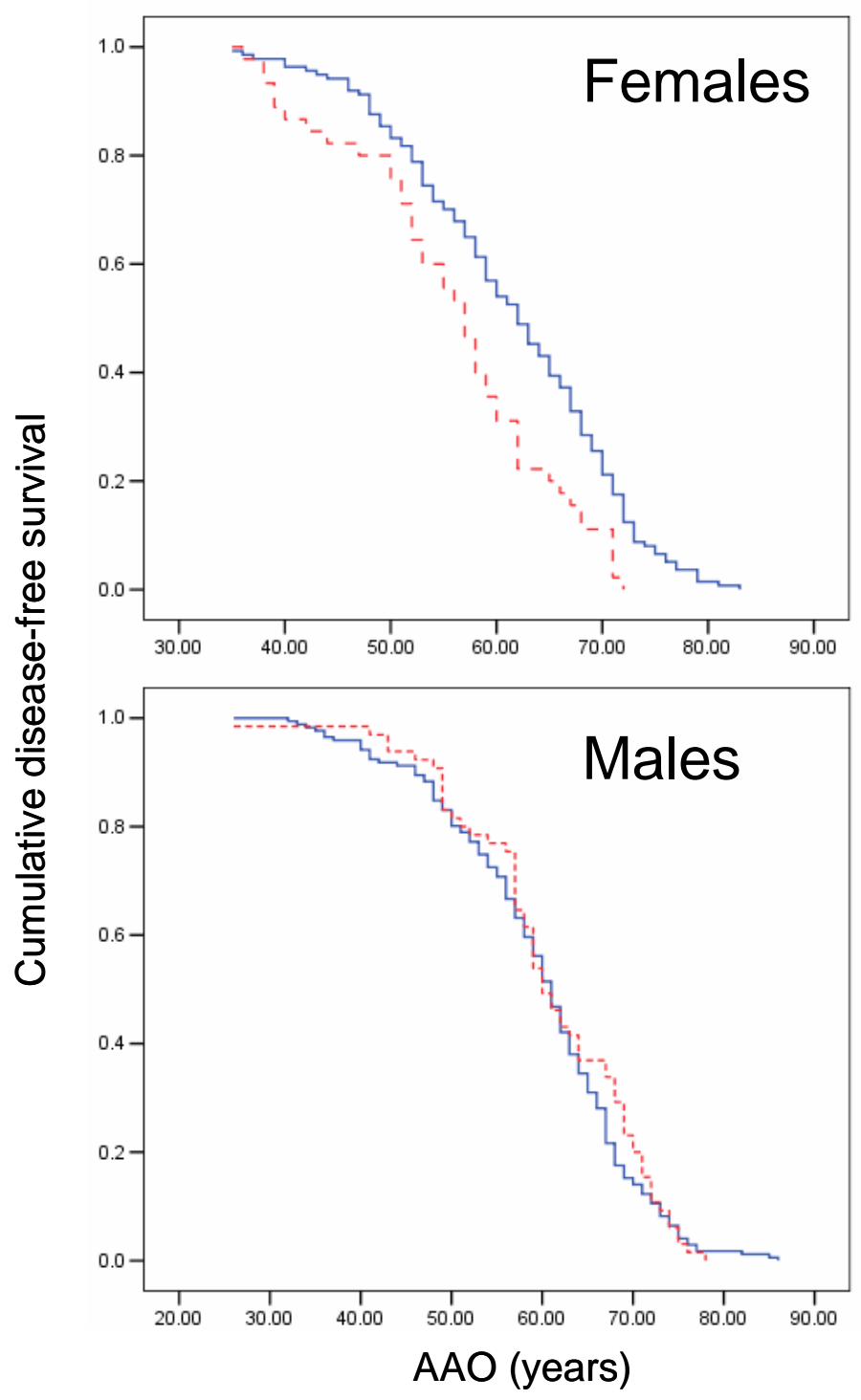




\section{REFERENCES}

1. Lohmueller KE, Pearce CL, Pike M, Lander ES, Hirschhorn JN. Meta-analysis of genetic association studies supports a contribution of common variants to susceptibility to common disease. Nat Genet 2003;33(2):177-182.

2. Masliah E, Rockenstein E, Veinbergs I, Sagara Y, Mallory M, Hashimoto M, et al. beta-amyloid peptides enhance alpha-synuclein accumulation and neuronal deficits in a transgenic mouse model linking Alzheimer's disease and Parkinson's disease. Proc Natl Acad Sci U S A 2001;98(21):12245-12250.

3. Blomqvist ME, Silburn PA, Buchanan DD, Andreasen N, Blennow K, Pedersen $\mathrm{NL}$, et al. Sequence variation in the proximity of IDE may impact age at onset of both Parkinson disease and Alzheimer disease. Neurogenetics 2004;5(2):115-119.

4. Martinez M, Brice A, Vaughan JR, Zimprich A, Breteler MM, Meco G, et al. Apolipoprotein E4 is probably responsible for the chromosome 19 linkage peak for Parkinson's disease. Am J Med Genet B Neuropsychiatr Genet 2005;136(1):72-74.

5. Huang X, Chen PC, Poole C. APOE-epsilon2 allele associated with higher prevalence of sporadic Parkinson disease. Neurology 2004;62(12):2198-2202.

6. Li YJ, Hauser MA, Scott WK, Martin ER, Booze MW, Qin XJ, et al. Apolipoprotein E controls the risk and age at onset of Parkinson disease. Neurology 2004;62(11):2005-2009.

7. Buchanan DD, Silburn PA, Chalk JB, Le Couteur DG, Mellick GD. The Cys282Tyr polymorphism in the HFE gene in Australian Parkinson's disease patients. Neurosci Lett 2002;327(2):91-94.

8. Mellick GD, Silburn PA, Prince JA, Brookes AJ. A novel screen for nuclear mitochondrial gene associations with Parkinson's disease. J Neural Transm 2004;111(2):191-199.

9. Whitbread AK, Mellick GD, Silburn PA, Le Couteur DG, Board PG. Glutathione transferase Omega class polymorphisms in Parkinson disease. Neurology 2004;62(10):1910-1911.

10. Zareparsi S, Camicioli R, Sexton G, Bird T, Swanson P, Kaye J, et al. Age at onset of Parkinson disease and apolipoprotein E genotypes. Am J Med Genet 2002;107(2):156-161.

11. Maraganore DM, Farrer MJ, Hardy JA, McDonnell SK, Schaid DJ, Rocca WA. Case-control study of debrisoquine 4-hydroxylase, $\mathrm{N}$-acetyltransferase 2, and apolipoprotein E gene polymorphisms in Parkinson's disease. Mov Disord 2000;15(4):714-719.

12. Corder EH, Ghebremedhin E, Taylor MG, Thal DR, Ohm TG, Braak H. The biphasic relationship between regional brain senile plaque and neurofibrillary tangle distributions: modification by age, sex, and APOE polymorphism. Ann N Y Acad Sci 2004;1019:24-28.

13. Ghebremedhin E, Schultz C, Thal DR, Rub U, Ohm TG, Braak E, et al. Gender and age modify the association between APOE and AD-related neuropathology. Neurology 2001;56(12):1696-1701. 